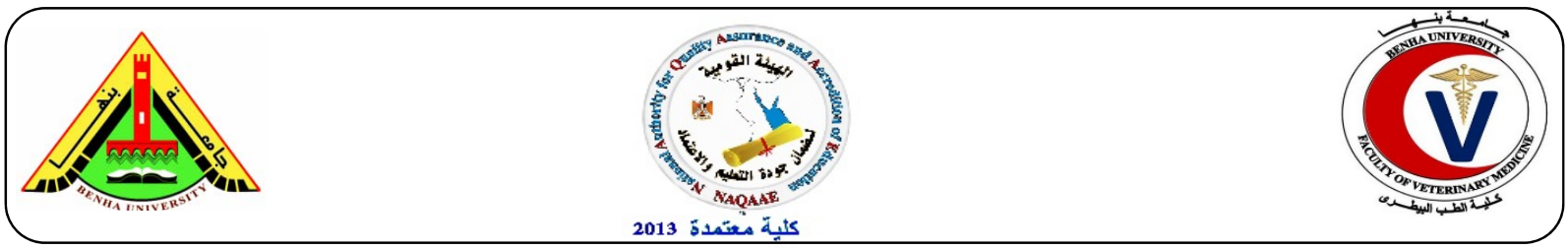

\title{
Screening the activity of anti-tuberculosis drugs against $M$. bovis BCG Connaught and $M$. bovis BCG Pasteur growing in J774A.1 cell line macrophages.
}

\author{
Ashraf A. Abd El-Tawab ${ }^{1}$, Fatma I. El-Hofy ${ }^{1}$, Essam A. Nasr ${ }^{2}$, Nammalwar Sriranganathan ${ }^{3}$, \\ Enas A. Soliman ${ }^{1}$ \\ Department of Bacteriology, Immunology and Mycology Fac. Vet. Med. Benha University, Egypt ${ }^{1,}$ \\ Department of diagnostic bacteriology. Veterinary serums and vaccines research Institute, Abbasia, Cairo, Egypt ${ }^{2}$. \\ Department of Biomedical Sciences \& Pathobiology Virginia-Maryland College of Veterinary Medicine, USA ${ }^{3}$.
}

\begin{abstract}
A B S T R A C T
Mycobacterium bovis BCG is attenuated strain derived from $M$. bovis. It has been known to prevent childhood pulmonary and meningeal tuberculosis and to treat superficial bladder cancer as immunotherapy. Administration of BCG has been restricted to immune-deficient individuals since 2007 because of its combined complications. Which treated with antituberculosis drugs based on assumption is fully responsive to these drugs. BCG Connaught showed more susceptibility to the activity of isoniazid, ethambutol, kanamycin, ofloxacin, streptomycin, amikacin and capreomycin than BCG Pasteur growing in J774A.1 cells. While the activity of rifampicin and levofloxacin was the same between the two tested strains. The spectrum of intracellular drug action can be ordered based on a decreasing order of inhibitory activity, as following rifampin $>$ isoniazid $>$ ethambutol $>$ streptomycin $>$ kanamycin $>$ amikacin $>$ ofloxacin $>$ capreomycin $>$ levofloxacin against BCG Connaught. While rifampin $>$ isoniazid $>$ ethambutol $>$ kanamycin $=$ streptomycin $>$ ofloxacin $>$ amikacin $>$ levofloxacin $>$ capreomycin for BCG Pasteur. There is a very limited publications describe the activity of antituberculosis drugs against intracellular growing BCG strains.
\end{abstract}

Keywords: $M$. bovis BCG - anti-tuberculosis drugs - macrophages.

(http://www.bvmj.bu.edu.eg)

(BVMJ-31(1): 124-131, 2016)

\section{INTRODUCTION}

Tuberculosis is a serious infectious bacterial disease caused by members of mycobacterium tuberculosis complex (MTBC) which includes M. tuberculosis, M. africanum, M. bovis, M. bovis BCG, M. microti, M. canettii, M. pinnipedii, M. mungi (Thoen et al., 2009). They are facultative intracellular pathogens, where they can survive and replicate within host macrophages through several mechanisms to escape macrophage destruction ability (Xu et al., 1994). These survival mechanisms make mycobacterial infections efficient and once intracellular they are difficult to treat by conventional antibiotics that do not accumulate well in the macrophage where the pathogen is sequestered (Barrow W, 2001). Therefore, evaluation of drug activity against intracellular growing mycobacterium is one of the main steps in screening drug susceptibility against mycobacterium (Andreu et al., 2012). M. bovis BCG is a member of MTBC. It is attenuated strain derived from M. bovis (Russell et al., 2010). $\mathrm{BCG}$ has been known to safely prevent severe form of the disease like childhood pulmonary and meningeal tuberculosis (Roy et al., 2014) and to treat superficial bladder cancer as immunotherapy (Watts et al., 2011 and Lukacs et al., 2013). But its administration is not recommended to individuals with impaired cellular immunity since 2007 because of its combined complications (FitzGerald, 2000; Paiman et al., 2006 and Arend and van Soolingen, 2011). Which may involve lifethreatening side effects including BCG sepsis (Lukacs et al., 2013). These complications combating regime depends on using antituberculosis drugs; with assumption of BCG is fully responsive to these drugs (Kolibab et al., 2011 and Fahimzad et al., 2015). To the best of authors knowledge there is a very limited literatures describe the susceptibility of antituberculosis drugs against intracellular growing BCG.

Therefore, this paper was aimed to screen the activity of nine antituberculosis drugs against two of common BCG strains growing in J774A.1 cell line macrophages. 
Screening the activity of anti-tuberculosis drugs against M. bovis BCG Connaught and M. bovis BCG Pasteur

\section{MATERIAL AND METHODS:}

\subsection{Mycobacterial strains:}

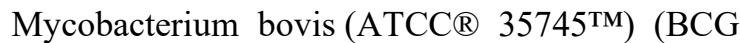
Connaught) and Mycobacterium bovis (ATCC $\mathbb{}$ $35734^{\mathrm{TM}}$ ) (BCG Pasteur) were obtained from American Type Culture Collection (ATCC, USA).

\subsection{Cell culture:}

According to manufacturer instructions, Mouse Macrophages J774A.1 (ATCC ${ }^{\circledR}$ TIB-67 $7^{\mathrm{TM}}$ ) was maintained in $75 \mathrm{~cm}^{2}$ flask containing $15 \mathrm{ml} 1 \mathrm{X}$ Dulbecco's modification of Eagle's Medium (DMEM) (Corning Cellgro, Mediatech, Inc. USA, Catalog No. 10-013-CV) supplemented with 10\% Heat in-activated Calf Serum-Iron fortified (FBS) (Sigma-Aldrich, Catalog No. $15 \mathrm{~A} 104$ ) at $37^{\circ} \mathrm{C}$ in humidified, $5 \% \quad \mathrm{CO}_{2}$ atmosphere until $90 \%$ confluent.

\subsection{Antimicrobial agents:}

Isoniazid (INH), streptomycin (SM), ethambutol (EMB), rifampicin (RMP), pyrazinamide (PZA), amikacin (AMI), ofloxacin (OFL), levofloxacin (LEV) and capreomycin (CAP) (Sigma, USA) and kanamycin (KAN) (Fisher Biotech). All the drugs were dissolved in sterile distilled water except RMP, OFL and LEV were dissolved in methyl alcohol, $1.0 \mathrm{~N}$ sodium hydroxide $(\mathrm{NaOH})$ and $0.1 \mathrm{~N} \mathrm{NaOH}$ respectively. They were kept in aliquot at $-20^{\circ} \mathrm{C}$ at final concentration of $10 \mathrm{mg} / \mathrm{L}$ until used (NCCLS, 2003).

\subsection{Drug concentrations:}

Three concentrations of each drug (Table 1) were used to treat infected macrophage cell line for two and five days. It was selected depending on previously determined $\mathrm{MBC}$ concentrations against BCG Connaught (data not published yet). The drug concentrations were diluted in DMEM supplemented with $1 \%$ FBS.

\subsection{MTS Cell Proliferation assay or Cytotoxicity assay (Rajasekaran et al., 2013)}

To determine whether the tested drug concentrations had any cytotoxic effect on macrophage cell line, a tetrazolium compound based cell viability assay [CellTiter 96® AQueous Non-Radioactive Cell Proliferation Assay (MTS assay) (Promega Inc., USA, Catalog No. G5421) was performed on J774A.1 macrophage. MTS is a tetrazolium dye that reduced by the live cell and produce water soluble colored formazan product. The macrophages were incubated with each drug concentration, in triplicate, overnight at $37^{\circ} \mathrm{C}$ under $5 \% \mathrm{CO}_{2}$. The number of the viable cells were measured based on the amount of absorbance at $490 \mathrm{~nm}$ due to formazan product. Cell viability present was calculated using this equation:

Average treatment data of each drug/avg control formazan data (un-treated) $\times 100$. The drug concentration was considered toxic if the cell viability below $90 \%$.

\subsection{Processing of BCG for infection (NCCLS, 2003 and Talaue et al., 2006):}

1 McFarland No. 1 standardized BCG suspension was prepared as described in M24-A of the Clinical and Laboratory Standards Institute (NCCLS, 2003). Then it was diluted (1:5) in phosphate-buffered saline (PBS) and centrifuged at $10,000 \mathrm{Xg}$ for $10 \mathrm{~min}$ at room temperature. The bacterial pellet was re-suspended in DMEM supplemented with $10 \%$ FBS.

\subsection{Macrophage assay (Rastogi and Blom-Potar, 1990; Talaue et al., 2006; Andreu et al., 2012 and Jhamb et al., 2014):}

J774A. 1 cells were seeded at a concentration of $1 \times 10^{4}$ cells/ well in 96-well tissue culture white

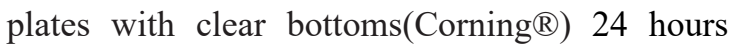
prior to infection (until the cells became spindle). After washing, the cells were infected with $100 \mu \mathrm{l}$ of prepared BCG suspension (multiplicity of infection, MOI, 1-10: 1). After 2 hours of infection at $37^{\circ} \mathrm{C}$ in $5 \% \mathrm{CO}_{2}$, macrophages were washed twice with HBSS, 1X (Hank's Balanced salt solution) (Cellgro ${ }^{\circledR}$ Mediatech, Inc. USA, Catalog No. 21-021-CV) to eliminate any extracellular bacteria. finally, $300 \mu 1$ of DMEM supplemented with $1 \%$ FBS, with or without drugs, was added to each well. The plates were incubated at $37^{\circ} \mathrm{C}$ in humidified $5 \% \quad \mathrm{CO}_{2}$ atmosphere for up to 5 days. The media with/without drugs were changed when the media began to show acidic reaction, orange color, (usually after 2 days). The cells were lysed, after time intervals 2 and 5 days, with SDS $0.125 \%$ for 20 minutes. The cell lysate was serially diluted and plated on Middlebrook 7H10 agar (Becton Dickinson and company, USA, Catalog No. 262710) supplemented with OADC $10 \%$ (Becton Dickinson and company, USA, Catalog No. 212351) for determination of colony forming units (CFUs). The experiment was repeated three times, each in triplicate.

\subsection{Statistical analysis:}

Unpaired, two tailed student's $t$ test was performed between the three sets of the experiment. A $p$ values of $\leq 0.05$ were considered significant. 


\section{RESULTS}

\subsection{Cytotoxicity assay:}

The cytotoxic effect of anti-tuberculosis drugs were determined using MTS assay. Figure 1 showed that all tested concentrations of the drugs were nontoxic. Except for levofloxacin at concentration $1 \mu \mathrm{g} / \mathrm{ml}$ showed fair cytopathic effect $(88.25 \%$ viable cell $)$

\subsection{Sensitivity of actively growing intracellular BCG against anti-tuberculosis drugs:}

The susceptibility of actively growing intracellular M. bovis (ATCC ${ }^{\circledR} 35745^{\mathrm{TM}}$ ) (BCG

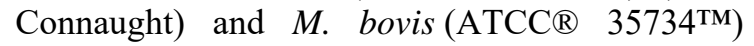
(BCG Pasteur) in J774A.1 cells to antituberculosis drugs after $2 \& 5$ days' treatment. The results were expressed as Log CFUs/ml \pm standard error, log reduction and present of inhibition compared with the growth of bacteria in the untreated cells (Table 2 \&3). BCG Connaught showed more susceptibility to the activity of isoniazid, ethambutol, kanamycin, ofloxacin, streptomycin, amikacin and capreomycin than BCG Pasteur growing in J774A.1 cells. While the activity of rifampicin and levofloxacin was the same between the two tested strains. The spectrum of intracellular drug action can be ordered based on a decreasing order of inhibitory activity, as following rifampin $>$ isoniazid $>$ ethambutol $>$ streptomycin $>$ kanamycin $>$ amikacin $>$ ofloxacin $>$ capreomycin $>$ levofloxacin against BCG Connaught. While rifampin $>$ isoniazid $>$ ethambutol $>$ kanamycin $=$ streptomycin $>$ ofloxacin $>$ amikacin $>$ levofloxacin> capreomycin for BCG Pasteur.

Table 1. Anti-tuberculosis drug concentrations used to treat infected macrophage cell line.

\begin{tabular}{llll}
\hline Drug & Concentration $(\mu \mathrm{g} / \mathrm{ml})$ & Drug & Concentration $(\mu \mathrm{g} / \mathrm{ml})$ \\
\hline INH & $1.00-2.00-4.00$ & KAN & $2.5-5.00-10.00$ \\
RMP & $0.125-0.25-0.50$ & OFL & $0.50-1.00-2.00$ \\
SM & $1.00-2.00-4.00$ & LEV & $0.25-0.50-1.00$ \\
EMB & $2.00-4.00-8.00$ & CAP & $1.25-2.5-5.00$
\end{tabular}

isoniazid (INH), streptomycin (SM), ethambutol (EMB), rifampicin (RMP), pyrazinamide (PZA), amikacin (AMI), ofloxacin (OFL), levofloxacin (LEV) and capreomycin (CAP) and kanamycin (KAN).

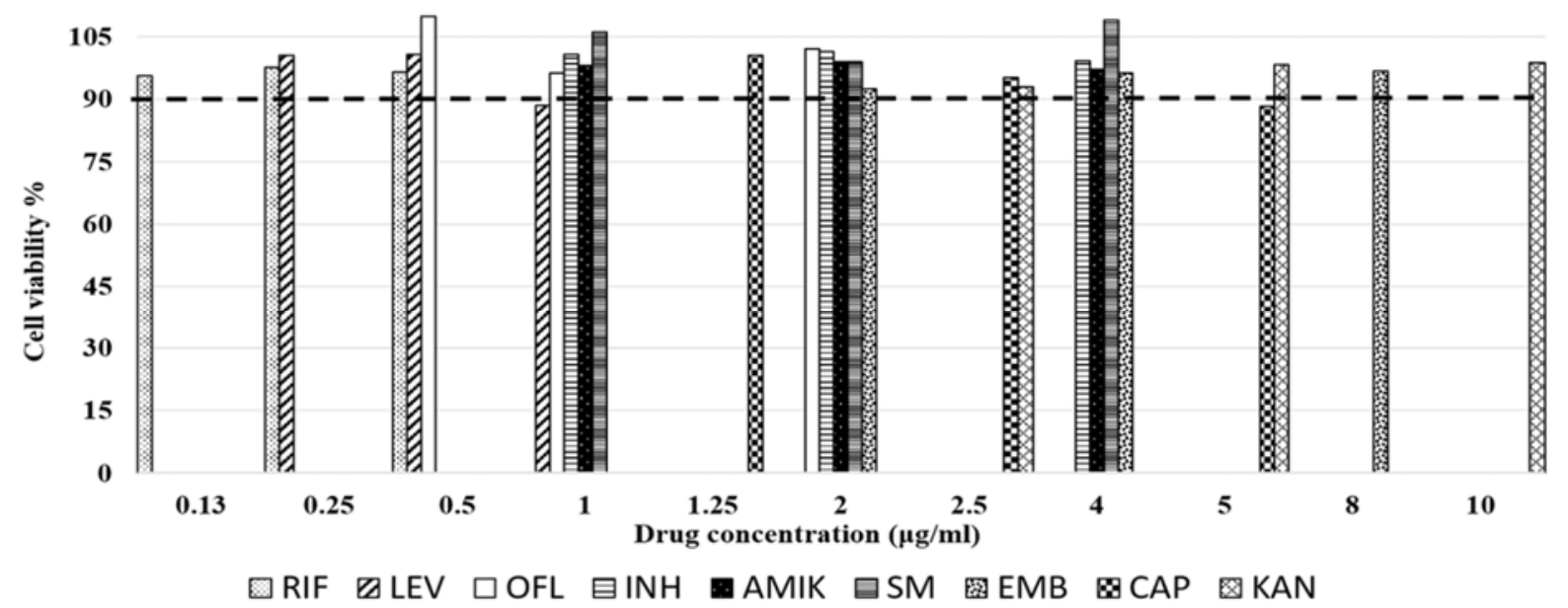

Figure 1: Cytotoxicity of anti-tuberculosis drugs on J774A.1 cells, the data represents the means of triplicate. The dotted line represented $90 \%$ of viable cell. 
Screening the activity of anti-tuberculosis drugs against M. bovis BCG Connaught and M. bovis BCG Pasteur

Table (2) susceptibility of $M$. bovis (ATCC $\AA 35745^{\mathrm{TM}}$ ) (BCG Connaught) to anti-tuberculosis drugs (MOI 110 CFU:1 macrophage)

\begin{tabular}{|c|c|c|c|c|c|c|c|c|c|c|}
\hline \multicolumn{11}{|c|}{ M. bovis (ATCC® $35745^{\mathrm{TM}}$ ) (BCG Connaught) } \\
\hline & \multicolumn{5}{|c|}{ Two days' treatment } & \multicolumn{5}{|c|}{ Five days' treatment } \\
\hline $\begin{array}{l}\text { Drug } \\
\text { conc. } \\
\mathrm{mg} / \mathrm{L}\end{array}$ & $\begin{array}{l}\text { Control } \\
\text { (untreated } \\
\text { ) Log } \\
\text { CFUs/m1* }\end{array}$ & $\begin{array}{c}\text { Drug } \\
\text { (treated) } \\
\text { Log } \\
\text { CFUs/ml } \\
*\end{array}$ & $\begin{array}{c}P \\
\text { value* } \\
*\end{array}$ & $\begin{array}{c}\log \\
\text { reductio } \\
\mathrm{n}\end{array}$ & $\begin{array}{c}\% \text { of } \\
\text { inhibitio } \\
n\end{array}$ & $\begin{array}{c}\text { Control } \\
\text { (untreated } \\
\text { ) Log } \\
\text { CFUs/m1* }\end{array}$ & $\begin{array}{c}\text { Drug } \\
\text { (treated) } \\
\text { Log } \\
\text { CFUs/ml* }\end{array}$ & $\begin{array}{c}P \\
\text { value* } \\
*\end{array}$ & $\begin{array}{c}\log \\
\text { reductio } \\
\mathrm{n}\end{array}$ & $\begin{array}{c}\% \text { of } \\
\text { inhibitio } \\
\mathrm{n}\end{array}$ \\
\hline \multicolumn{11}{|l|}{ INH } \\
\hline 1 & & $6.16 \pm 0.36$ & 0.03 & 0.54 & 65.24 & \multirow{3}{*}{$7.65 \pm 0.08$} & $6.23 \pm 0.09$ & 0.00 & 1.42 & 96.14 \\
\hline 2 & & $6.17 \pm 0.38$ & 0.04 & 0.53 & 63.45 & & $6.11 \pm 0.07$ & 0.00 & 1.54 & 97.14 \\
\hline 4 & & $6.08 \pm 0.36$ & 0.02 & 0.61 & 70.77 & & $6.04 \pm 0.02$ & 0.00 & 1.61 & 97.63 \\
\hline RIF & \multirow[t]{4}{*}{$6.7 \pm 0.27$} & & & & & & & & & \\
\hline $\begin{array}{c}0.12 \\
5\end{array}$ & & $6.15 \pm 0.23$ & 0.00 & 0.55 & 73.41 & \multirow[t]{3}{*}{$7.53 \pm 0.08$} & $6.49 \pm 0.11$ & 0.00 & 1.04 & 90.57 \\
\hline 0.25 & & $5.84 \pm 0.26$ & 0.00 & 0.86 & 86.24 & & $5.83 \pm 0.20$ & 0.00 & 1.70 & 97.60 \\
\hline 0.50 & & $5.56 \pm 0.23$ & 0.00 & 1.14 & 93.21 & & $5.45 \pm 0.09$ & 0.00 & 2.08 & 99.17 \\
\hline \multicolumn{11}{|l|}{ SM } \\
\hline 1 & \multirow{3}{*}{$7.05 \pm 0.08$} & $6.84 \pm 0.13$ & 0.05 & 0.18 & 34.89 & \multirow{3}{*}{$7.50 \pm 0.03$} & $7.32 \pm 0.01$ & 0.00 & 0.26 & 35.22 \\
\hline 2 & & $6.78 \pm 0.14$ & 0.05 & 0.23 & 41.23 & & $7.12 \pm 0.01$ & 0.00 & 0.45 & 58.84 \\
\hline 4 & & $6.70 \pm 0.13$ & 0.02 & 0.31 & 52.18 & & $\begin{array}{c}6.97 \pm 0.0 .0 \\
3\end{array}$ & 0.00 & 0.64 & 70.55 \\
\hline \multicolumn{11}{|l|}{ EMB } \\
\hline 2 & \multirow[t]{3}{*}{$6.86 \pm 0.35$} & $6.36 \pm 0.30$ & 0.01 & 0.49 & 71.25 & \multirow[t]{3}{*}{$7.79 \pm 0.01$} & $7.14 \pm 0.01$ & 0.00 & 0.65 & 77.82 \\
\hline 4 & & $6.19 \pm 0.33$ & 0.00 & 0.66 & 79.10 & & $6.83 \pm 0.002$ & 0.00 & 0.97 & 89.28 \\
\hline 8 & & $6.08 \pm 0.40$ & 0.01 & 0.77 & 80.83 & & $6.48 \pm 0.02$ & 0.00 & 1.32 & 95.18 \\
\hline \multicolumn{11}{|l|}{ CAP } \\
\hline 1.25 & \multirow[t]{3}{*}{$6.69 \pm 0.26$} & $6.66 \pm 0.23$ & 0.41 & 0.04 & 14.22 & \multirow[t]{3}{*}{$7.71 \pm 0.04$} & $7.75 \pm 0.03$ & 0.47 & -0.04 & -9.60 \\
\hline 2.5 & & $6.65 \pm 0.22$ & 0.41 & 0.05 & 16.39 & & $7.56 \pm 0.00$ & 0.02 & 0.15 & 29.97 \\
\hline 5 & & $6.62 \pm 0.24$ & 0.16 & 0.07 & 19.05 & & $7.38 \pm 0.00$ & 0.00 & 0.33 & 53.67 \\
\hline \multicolumn{11}{|l|}{$\mathrm{AM}$} \\
\hline 0.50 & \multirow{3}{*}{$5.95 \pm 0.50$} & $5.94 \pm 0.51$ & 0.07 & 0.01 & 1.57 & \multirow{3}{*}{$7.78 \pm 0.03$} & $7.55 \pm 0.01$ & 0.00 & 0.24 & 42.46 \\
\hline 1.00 & & $5.93 \pm 0.51$ & 0.19 & 0.02 & 0.85 & & $7.42 \pm 0.01$ & 0.00 & 0.36 & 56.78 \\
\hline 2.00 & & $5.84 \pm 0.54$ & 0.08 & 0.12 & 11.28 & & $7.22 \pm 0.02$ & 0.00 & 0.56 & 72.82 \\
\hline \multicolumn{11}{|l|}{ KAN } \\
\hline 2.5 & \multirow{3}{*}{$7.10 \pm 0.14$} & $7.06 \pm 0.12$ & 0.25 & 0.04 & 12.48 & \multirow{3}{*}{$7.55 \pm 0.06$} & $7.24 \pm 0.05$ & 0.01 & 0.12 & 51.44 \\
\hline 5.00 & & $7.02 \pm 0.12$ & 0.11 & 0.08 & 19.89 & & $7.06 \pm 0.04$ & 0.00 & 0.44 & 68.06 \\
\hline $\begin{array}{c}10.0 \\
0\end{array}$ & & $6.93 \pm 0.15$ & 0.03 & 0.17 & 31.58 & & $6.82 \pm 0.05$ & 0.00 & 0.68 & 81.30 \\
\hline \multicolumn{11}{|l|}{ LEV } \\
\hline 0.25 & \multirow[t]{3}{*}{$7.04 \pm 0.18$} & $7.10 \pm 0.19$ & 0.01 & -0.06 & -15.06 & $7.81 \pm 0.01$ & $7.77 \pm 0.01$ & 0.06 & 0.04 & 8.98 \\
\hline 0.50 & & $7.10 \pm 0.21$ & 0.17 & -0.06 & -21.64 & & $7.75 \pm 0.01$ & 0.01 & 0.06 & 13.95 \\
\hline 1.00 & & $7.04 \pm 0.21$ & 0.94 & 0.00 & -6.10 & & $7.62 \pm 0.01$ & 0.00 & 0.19 & 35.75 \\
\hline OFL & & & & & & & & & & \\
\hline 0.5 & $645+050$ & $6.52 \pm 0.48$ & 0.10 & -0.07 & -12.84 & $7.73 \pm 0.03$ & $7.69 \pm 0.01$ & 0.15 & 0.05 & 10.92 \\
\hline 1 & $0.45 \pm 0.50$ & $6.46 \pm 0.49$ & 0.86 & 0.00 & 2.76 & & $7.51 \pm 0.01$ & 0.00 & 0.22 & 40.61 \\
\hline 2 & & $6.43 \pm 0.51$ & 0.10 & 0.02 & 3.20 & & $7.24 \pm 0.01$ & 0.00 & 0.49 & 67.97 \\
\hline
\end{tabular}

$*$ Mean \pm standard error in three different experiments. ${ }^{* *} p$ value of $\leq 0.05$ was considered statistically significant using unpaired, two tailed student's $t$ test. 
Table (3) susceptibility of M. bovis (ATCC® $35734^{\mathrm{TM}}$ ) (BCG Pasteur) to antituberculosis drugs (MOI, 3-10 CFUs:1 macrophage)

M. bovis (ATCC® $35734^{\mathrm{TM}}$ ) (BCG Pasteur)

\begin{tabular}{|c|c|c|c|c|c|c|c|c|c|c|}
\hline \multicolumn{6}{|c|}{ Two days' treatment } & \multicolumn{5}{|c|}{ Five days' treatment } \\
\hline $\begin{array}{l}\text { Drug } \\
\text { conc. } \\
\mathrm{mg} / \mathrm{L}\end{array}$ & $\begin{array}{c}\text { Control } \\
\text { (untreated) } \\
\text { Log } \\
\text { CFUs/ml* }\end{array}$ & $\begin{array}{c}\text { Drug } \\
\text { (treated) } \\
\text { Log } \\
\text { CFUs } / \mathrm{ml}^{*}\end{array}$ & $\begin{array}{c}P \\
\text { value } * *\end{array}$ & $\begin{array}{l}\log \\
\text { reduction }\end{array}$ & $\begin{array}{c}\% \text { of } \\
\text { inhibition }\end{array}$ & $\begin{array}{c}\text { Control } \\
\text { (untreated) } \\
\text { Log } \\
\text { CFUs } / \mathrm{ml}^{*}\end{array}$ & $\begin{array}{c}\text { Drug } \\
\text { (treated) } \\
\text { Log } \\
\text { CFUs/m* }\end{array}$ & $\begin{array}{c}P \\
\text { value** }\end{array}$ & $\begin{array}{l}\log \\
\text { reduction }\end{array}$ & $\begin{array}{c}\% \text { of } \\
\text { inhibition }\end{array}$ \\
\hline \multicolumn{11}{|l|}{ INH } \\
\hline 1 & & $4.04 \pm 0.03$ & 0.01 & 0.52 & 71.45 & \multirow{3}{*}{$6.72 \pm 0.04$} & $6.19 \pm 0.01$ & 0.00 & 0.53 & 70.65 \\
\hline 2 & $4.56 \pm 0.11$ & $3.81 \pm 0.03$ & 0.00 & 0.75 & 83.19 & & $6.15 \pm 0.01$ & 0.00 & 0.57 & 73.17 \\
\hline 4 & & $3.90 \pm 0.05$ & 0.01 & 0.66 & 78.99 & & $6.22 \pm 0.02$ & 0.00 & 0.51 & 68.97 \\
\hline \multicolumn{11}{|l|}{ RIF } \\
\hline 0.125 & $6.62 \pm 0.004$ & $6.12 \pm 0.03$ & 0.00 & 0.50 & 68.49 & \multirow{3}{*}{$5.92 \pm 0.02$} & $4.95 \pm 0.02$ & 0.00 & 0.97 & 89.18 \\
\hline 0.25 & & $5.91 \pm 0.04$ & 0.00 & 0.71 & 80.54 & & $4.71 \pm 0.03$ & 0.00 & 1.21 & 93.79 \\
\hline 0.50 & & $5.45 \pm 0.03$ & 0.00 & 1.17 & 93.25 & & $4.49 \pm 0.01$ & 0.04 & 1.43 & 96.30 \\
\hline \multicolumn{11}{|l|}{ SM } \\
\hline 1 & $5.93 \pm 0.02$ & $5.90 \pm 0.01$ & 0.24 & 0.03 & 7.02 & \multirow[t]{3}{*}{$6.21 \pm 0.04$} & $6.15 \pm 0.02$ & 0.23 & 0.06 & 12.93 \\
\hline 2 & & $5.87 \pm 0.01$ & 0.07 & 0.06 & 12.48 & & $6.05 \pm 0.02$ & 0.02 & 0.16 & 30.61 \\
\hline 4 & & $5.74 \pm 0.02$ & 0.00 & 0.19 & 34.89 & & $5.95 \pm 0.0 .03$ & 0.01 & 0.26 & 45.58 \\
\hline \multicolumn{11}{|l|}{ EMB } \\
\hline 2 & $4.56 \pm 0.11$ & $3.90 \pm 0.08$ & 0.01 & 0.65 & 78.41 & \multirow[t]{3}{*}{$6.72 \pm 0.04$} & $6.37 \pm 0.04$ & 0.00 & 0.35 & 55.03 \\
\hline 4 & & $4.10 \pm 0.07$ & 0.03 & 0.45 & 65.94 & & $6.21 \pm 0.002$ & 0.00 & 0.51 & 69.08 \\
\hline 8 & & $4.01 \pm 0.02$ & 0.01 & 0.54 & 73.04 & & $6.16 \pm 0.02$ & 0.00 & 0.56 & 72.43 \\
\hline \multicolumn{11}{|l|}{ CAP } \\
\hline 1.25 & $5.04 \pm 0.03$ & $4.96 \pm 0.06$ & 0.25 & 0.09 & 17.04 & \multirow[t]{3}{*}{$4.84 \pm 0.01$} & $4.86 \pm 0.03$ & 0.72 & -0.01 & -2.86 \\
\hline 2.5 & & $4.99 \pm 0.05$ & 0.25 & 0.06 & 11.39 & & $4.69 \pm 0.05$ & 0.03 & 0.15 & 28.57 \\
\hline 5 & & $4.92 * * *$ & & 0.13 & 25.69 & & $4.74 \pm 0.03$ & 0.04 & 0.10 & 20.95 \\
\hline \multicolumn{11}{|l|}{$\mathrm{AM}$} \\
\hline 0.50 & & $5.05 \pm 0.00$ & 0.92 & 0.00 & -0.25 & \multirow{3}{*}{$6.25 \pm 0.04$} & $6.21 \pm 0.01$ & 0.38 & 0.04 & 9.03 \\
\hline 1.00 & $5.04 \pm 0.03$ & $4.99 \pm 0.01$ & 0.19 & 0.05 & 11.72 & & $6.19 \pm 0.01$ & 0.19 & 0.06 & 13.40 \\
\hline 2.00 & & $4.87 \pm 0.02$ & 0.01 & 0.18 & 33.67 & & $6.07 \pm 0.01$ & 0.01 & 0.18 & 33.96 \\
\hline \multicolumn{11}{|l|}{ KAN } \\
\hline 2.5 & $5.04 \pm 0.03$ & $4.87 \pm 0.05$ & 0.03 & 0.17 & 32.67 & \multirow{3}{*}{$6.25 \pm 0.04$} & $6.16 \pm 0.01$ & 0.08 & 0.09 & 19.00 \\
\hline 5.00 & & $4.96 \pm 0.01$ & 0.06 & 0.09 & 18.70 & & $6.13 \pm 0.01$ & 0.03 & 0.12 & 24.61 \\
\hline 10.00 & & $4.88^{* * *}$ & & 0.17 & 32.67 & & $5.99 \pm 0.02$ & 0.00 & 0.25 & 44.55 \\
\hline \multicolumn{11}{|l|}{ LEV } \\
\hline 0.25 & $5.97 \pm 0.02$ & $5.98 \pm 0.06$ & 0.81 & -0.02 & -5.11 & \multirow{3}{*}{$6.21 \pm 0.04$} & $6.16 \pm 0.02$ & 0.26 & 0.06 & 12.24 \\
\hline 0.50 & & $5.99 \pm 0.03$ & 0.58 & -0.02 & -5.11 & & $6.3 \pm 0.08$ & 0.41 & 0.08 & 14.97 \\
\hline 1.00 & & $6.03 \pm 0.04$ & 0.21 & -0.06 & -16.52 & & $6.06 \pm 0.03$ & 0.03 & 0.15 & 28.57 \\
\hline \multicolumn{11}{|l|}{ OFL } \\
\hline 0.5 & $5.04 \pm 0.03$ & $5.01 \pm 0.00$ & 0.29 & 0.04 & 8.73 & $6.25 \pm 0.04$ & $6.12 \pm 0.02$ & 0.03 & 0.21 & 26.48 \\
\hline 1 & & $4.94 \pm 0.03$ & 0.08 & 0.10 & 21.20 & & $6.12 \pm 0.01$ & 0.03 & 0.21 & 26.17 \\
\hline 2 & & $5.03 \pm 0.02$ & 0.65 & 0.02 & 4.24 & & $6.04 \pm 0.01$ & 0.00 & 0.21 & 38.94 \\
\hline
\end{tabular}

$*$ Mean \pm standard error in three different experiments. ** $p$ value of $\leq 0.05$ was considered statistically significant using unpaired, two tailed student's $t$ test. ${ }^{* * *}$ The Mean of three different wells in one experiment.

\section{DISCUSSION:}

Evaluation of drug activity against intracellular growing mycobacterium is not only important to determine ability of the drug to accumulate inside the macrophages but also to ensure that the drug is effective intracellularly. As the growth of the mycobacteria in the host has been observed to be quite different from its growth in vitro. So that upregulation of mycobacterial genes may be differed between both systems. The drug target (e.g. an enzyme in a key pathway) that may be upregulated in vitro may not be present at the same level or may not be expressed at all when the mycobacteria grow intracellularly. Which may give different drug profile against extracellular and intracellular mycobacterium (Barrow, 2001). The current study showed the activity of nine of most 
common antituberculosis drugs against intracellular growing BCG Connaught and BCG Pasteur strains.

INH was found to be the second drug after RIF to be active against both BCG strains. However, after 5 days' treatment, its inhibitory effect against intracellular BCG Connaught (up to 97.6\%) was more than those against intracellular BCG Pasteur strain (up to 73.2). which may indicate that $\mathrm{BCG}$ Pasteur strain had less susceptibility to INH than BCG Connaught, intracellularly, even in its obtainable serum level in human $(4 \mu \mathrm{g} / \mathrm{ml})$. Comparing to the MTB susceptibility to INH, BCG Connaught seemed to be quite similar to MTB and M. bovis, while BCG Pasteur was less susceptible. The previously published results showed that INH was highly effective against intracellular MTB as it inhibited more than $99 \%$ at $0.20 \mu \mathrm{g} / \mathrm{ml}$ after 7 days' treatment (Wright et al., 1996 and Jhamb et al., 2014). In addition, INH obtainable serum level in human $(4 \mu \mathrm{g} / \mathrm{ml})$ caused $97.6 \% \& 99.2 \%$ inhibition of MTB and M. bovis inside J774 cells after 5 days' treatment, respectively (Rastogi et al., 1987) or $99.65 \%$ after 7 days (Rastogi et al., 1996). Also it was reported that INH inhibited the mycobacterial growth at $0.05 \mu \mathrm{g} / \mathrm{ml}$ (Brennan et al., 2008).

RIF showed the most potent effective drugs against the intracellular BCG strains in the current study. Its activity against both BCG strains was quite the same. Comparing to its activity against MTB, it seemed to be higher against BCG strains. in this respect Rastogi et al. (1987) stated that 0.1 $\mu \mathrm{g} / \mathrm{ml}$ RIF inhibited $55 \%$ of MTB growing in J774 cells after 5 days' treatment. And when the cells treated with RIF serum level $(15 \mu \mathrm{g} / \mathrm{ml})$ there was $99.06 \%$ inhibition. In the current study, 0.125 inhibited 89.2-90.6 of both BCG strains tested and $0.5 \mu \mathrm{g} / \mathrm{ml}$ inhibited $99.2 \%$ of BCG Connaught and $96.3 \%$ of BCG Pasteur after 5 days.

There was another study that treated MTB growing in J774 and human MM6 cells with 0.016 $\mu \mathrm{g} / \mathrm{ml}$ ( 3 fold higher than MIC). and it has been found that it inhibited $95 \%$ of MTB growing in $\mathrm{J} 774$ cells and $99.5 \%$ of that growing in MM6 cells after 7 days of treatment (Wright et al., 1996). Additionally, it was found that RIF serum level inhibited about $99.99 \%$ of MTB growing in human macrophages after 7 days' treatment (Rastogi et al., 1996).

As presented in table $2 \& 3$, EMB was had significant growth inhibition on both BCG strains after 2 and 5 days of drug addition. This inhibition was more obvious against BCG Connaught after 5 days' treatment that showed exposure dependent inhibition (time $\mathrm{x}$ concentration). While $\mathrm{BCG}$ Pasteur growth inhibition was quite the same between the two-time point of cells lysis.

Previously, 7 days' treatment with $4 \mu \mathrm{g} / \mathrm{ml}$ EMB to J774 cells and human MM6 cells infected with MTB caused 96.98 and $99.2 \%$ growth inhibition respectively (Wright et al., 1996). Comparing to our results $4 \mu \mathrm{g} / \mathrm{ml}$ EMB activity against BCG Connaught (89.28 \% inhibition after 5 days) were more close to MTB than that of BCG Pasteur (69.1\%).

Another study tested the EMB serum level (6 $\mu \mathrm{g} / \mathrm{ml}$ ) against MBT in human macrophages. The growth inhibition was around $86.7 \%$ after 3 days and around $98.8 \%$ after 7 days (Rastogi et al., 1996). In the recent study, $8 \mu \mathrm{g} / \mathrm{ml}$ caused 80.8 95.18 inhibition to BCG Connaught after 2-5 days of drug addition, respectively. While BCG Pasteur growth was inhibited by 73.04-72.4 after 2-5 days' treatment.

The highest tested concentration of SM (4 $\mu \mathrm{g} / \mathrm{ml})$, as can be seen in table $2 \& 3$, inhibited $52.2 \%$ of BCG Connaught growth after 2 days of treatment and increased up to $70.6 \%$ after 5 days. While BCG Pasteur was inhibited by $34.9 \%$ and $45.6 \%$ after 2 and 5 days, respectively. This indicating that $\mathrm{SM}$ was more active against $\mathrm{BCG}$ Connaught than BCG Pasteur.

It has been found that $\mathrm{SM}$ at $1 \mu \mathrm{g} / \mathrm{ml}$ had no inhibition on MBT gowning inside $\mathrm{J} 774$ cells after 2 or 5 days' treatment. While $10 \mu \mathrm{g} / \mathrm{ml}$ inhibited only $50 \%$ of mycobacterial load after 5 days of treatment (Rastogi et al., 1987). Comparing to the current obtained results BCG Connaught was more susceptible to SM than BCG Pasteur and MTB.

In the current study AMK and KAN were also found to be more active against BCG Connaught than BCG Pasteur after 5 days' treatment. KAN at $10 \mu \mathrm{g} / \mathrm{ml}$ inhibited BCG Connaught and BCG Pasteur by $31.6-81.3 \%$ and $32.7-44.6 \%$ after 2 and 5 days of treatment, respectively. Previously, it has been found that the same concentration inhibited MTB growing in human THP1 cells by $44-58 \%$ after 3-7 days of treatment, respectively (Giovagnoli et al., 2013). While their serum level (KAN $30 \mu \mathrm{g} / \mathrm{ml}$ and AMI $20 \mu \mathrm{g} / \mathrm{ml}$ ) caused $90 \%$ and close to $99 \%$ killing of $M$. tuberculosisgrowing in macrophages (Brennan et al., 2008).

The inhibitory effect of CAP against both BCG strains was found to be accepted statistically $(P \leq 0.05)$ only at 2.5 and $5 \mu \mathrm{g} / \mathrm{ml}$ after 5 days of drug addition. $2.5 \mu \mathrm{g} / \mathrm{ml}$ cause nearly the same percent of growth inhibition of both strains (28- 
$30 \%$ ). While $5 \mu \mathrm{g} / \mathrm{ml}$ inhibited $53.7 \%$ of $\mathrm{BCG}$ Connaught and $20.95 \%$ of BCG Pasteur.

The CAP activity against MBT had been evaluated in human macrophages. At $10 \mu \mathrm{g} / \mathrm{ml}$, there was $63-88 \%$ inhibition after 3-7 days of treatment, respectively (Giovagnoli et al., 2013). And at $30 \mu \mathrm{g} / \mathrm{ml}$, there was around $75-95 \%$ inhibition after 3-7 days of treatment, respectively (Rastogi et al., 1996).

OFL showed no activity against both BCG strains after 2 days' treatment. While after 5 days' treatment the maximum inhibition was $67.97 \%$ of BCG Connaught and 38.9 for BCG Pasteur strain at concentration $2 \mu \mathrm{g} / \mathrm{ml}$. Comparing to intracellular MTB susceptibility to OFL, it had been reported that OFL at $2 \mu \mathrm{g} / \mathrm{ml}$ inhibited MTB growing in human THP1 cells by $40 \%$ and up to $100 \%$ after 3 and 7 days after treatment, respectively (Giovagnoli et al., 2013). Another study reported that OFL serum level $(5 \mu \mathrm{g} / \mathrm{ml})$ cause more than $99 \%$ inhibition of MBT gowning inside $\mathrm{J} 774$ cells or human macrophages after 5-7 days of treatment. And more than $90 \%$ inhibition when compared to initial bacterial count (just before drug addition) giving indication that OFL has bactericidal activity against MTB after 5 days (Rastogi and Blom-Potar, 1990 and Rastogi et al., 1996).

Depending on exposure dependent inhibition (time $\mathrm{x}$ concentration) of OFL against tested BCG strains may indicate that the susceptibility of BCG Connaught is closer to that of MTB than BCG Pasteur. And the intracellular susceptibility of both BCG strains to OFL was different.

LEV also showed no activity against both BCG strains after 2 days' treatment. Even after 5 days' treatment the maximum inhibition at $1 \mu \mathrm{g} / \mathrm{ml}$ was $35.7 \%$ and $28.57 \%$ for BCG Connaught and BCG Pasteur, respectively. The intracellular susceptibility of both BCG strains to LEV was quite similar to each other. But absolutely different from MTB as it was reported that LEV was active against $M$. tuberculosis-infected macrophages at $0.5 \mu \mathrm{g} / \mathrm{ml}$ (Brennan et al., 2008). previous study showed that LEV was had bactericidal effect against M. tuberculosis-infected $\mathrm{J} 7441$ cells at concentration of 1.547 and 0.878 to $1.708 \mu \mathrm{g} / \mathrm{ml}$ after 4 and 7 days' treatment, respectively (Andreu et al., 2012).

In conclusion: BCG Connaught showed more susceptibility to the activity of isoniazid, ethambutol, kanamycin, ofloxacin, streptomycin, amikacin and capreomycin than BCG Pasteur growing in J774A.1 cells. This primary screening of intracellular antituberculosis drug profile showed difference between two of BCG daughter strains and previously publish results against MTB. The serum level investigation of all antituberculosis drugs and their combination against all BCG daughter strains is recommended. To determine the most effective drug regime to be used for treatment of BCG complications. Which will help in avoiding of emergence of drug resistance BCG.

\section{REFERENCES:}

Andreu, N., Fletcher, T., Krishnan, N., Wiles, S. and Robertson, B.D. 2012. Rapid measurement of antituberculosis drug activity in vitro and in macrophages using bioluminescence. J Antimicrob Chemother, 67, 404-414.

Arend, M. and van Soolingen, D. 2011. Low Level INH-Resistant BCG: A Sheep in Wolf's Clothing? Clin Infect Dis, 52 (1), 89-93.

Barrow, WW. 2001. Treatment of mycobacterial infections. Rev. sci. tech. Off. int. Epiz., 20 (1), 55-70.

Brennan, P.J., Young, D.B. and Robertson, B.D. (Co-Editors and Deputy Editor). (2008). Handbook of Anti-Tuberculosis Agents. Global Alliance for TB Drug Development. Tuberculosis, 88. CElsevier Ltd.

Fahimzad, S.A., Ghasemi, M., Shiva, F., Ghadiri, K., Navidinia, M., Karimi A. 2015. Susceptibility Pattern of Bacille CalmetteGuerin Strains Against Pyrazinamide and Other Major Anti-Mycobacterial Drugs. Arch Pediatr Infect Dis, 3(1 TB), e17814.

FitzGerald, J.M. 2000. Management of Adverse Reactions to Bacille Calmette-Gue'rin Vaccine. Clinical Infectious Diseases, 31(Suppl 3), S75-6.

Giovagnoli, S., Marenzoni, M.L., Nocchetti, M., Santi, C., Blasi, P., Schoubben, A. and Ricci, M. 2013. Synthesis, characterization and in vitro extracellular and intracellular activity against Mycobacterium tuberculosis infection of new second-line antitubercular drug-palladium complexes. Journal of Pharmacy and Pharmacology, 66, 106-121.

Jhamb, S., Goyal, A. and Singh, P.P. 2014. Determination of the activity of standard anti-tuberculosis drugs against intramacrophage Mycobacterium tuberculosis, in vitro: MGIT 960 as a viable alternative for BACTEC 460. Braz J Infect Dis., 1 8(3), 336-340. 
Kolibab, K., Derrick, S.C., Morris, S.L. 2011. Sensitivity to isoniazid of Mycobacterium bovis BCG strains and BCG disseminated disease isolates. Journal of clinical microbiology, 2380-2381.

Lukacs, S., Tschobotko, B., Szabo, N.A. and Symes, A. 2013. Systemic BCG-Osis as a Rare Side Effect of Intravesical BCG Treatment for Superficial Bladder Cancer. Case Reports in Urology, Article ID 821526, 3 pages.

NCCLS. 2003. National Committee for Clinical Laboratory Standards. Susceptibility testing of Mycobacterium tuberculosis, Nocardiae, and other aerobic Actinomycetes: approved standard M-24A. Wayne, PA, USA.

Paiman, S.A., Siadati, A., Mamishi, S., Tabatabaie, P. and Khotaee, G. 2006. Disseminated Mycobacterium bovis Infection after BCG Vaccination. Iran $\mathbf{J}$ Allergy Asthma Immunol, 5,133-137.

Rajasekaran, P. Alexander, J.C., Seleem, M.N., Jain, N., Sriranganathan, N., Wattam, A.R., Setubal, J.C., Boyle, S.M. 2013. Peptide nucleic acids inhibit growth of Brucella suis in pure culture and in infected murine macrophages. International Journal of Antimicrobial Agents, 41, 358-362.

Rastogi, N. and Blom-Potar, M.C. 1990. Intracellular Bactericidal Activity of Ciprofloxacin and Ofloxacin Against Mycobacterium tuberculosis H37Rv Multiplying in the J-774 Macrophage Cell Line. Zbl. Bakt., 273, 195-199.

Rastogi, N., Labrousse, V. and Goh, K.S. 1996. In Vitro Activities of Fourteen Antimicrobial Agents Against Drug Susceptible and Resistant Clinical Isolates of Mycobacterium tuberculosis and Comparative Intracellular Activities Against the Virulent H37Rv Strain in Human Macrophages. Current Microbiology, 33, 167-175.

Rastogi, N., Potar, M. and David, H.L. 1987. Intracellular Growth of Pathogenic Mycobacteria in the Continuous Murine Macrophage Cell Line J-774: Ultrastructure and Drug-susceptibility Studies. Current microbiology, 16, 79-92.

Roy, A., Eisenhut, M., Harris, R.J., Rodrigues, L.C., Sridhar, S., Habermann, S., Snell, L., Mangtani, P., Adetifa, I., Lalvani, A. and Abubakar, I. 2014. Effect of BCG vaccination against Mycobacterium tuberculosis infection in children: systematic review and meta-analysis. BMJ, 349, g4643.

Russell, D.G., Barry, C.E. and Flynn, J.L. 2010. Tuberculosis: What We Don't Know Can, and Does, Hurt Us. Science., 328, 852-856.

Talaue, M.T., Venketaraman, V., Hazbo'n, M.H., Peteroy-Kelly, M., Seth, A., Colangeli, R., Alland, D. and Connell, N.D. 2006. Arginine Homeostasis in J774.1 Macrophages in the Context of Mycobacterium bovis BCG Infection. Journal of Bacteriology, 188, 4830-4840.

Thoen, C.O., LoBue, P.A., Enarson, D.A., Kaneene, J.B. and Kantor, I.N. 2009. tuberculosis: a re-emerging disease in animals and hmans. Veterinaria Italiana, 45(1), 135-181

Watts, M.R., Taylor, P.C., Sintchenko, V., Whelan, C.M., Farnsworth, R.H., Jones, P., Jelfs, P., Carr, D. and Goldberg, H. 2011. Implications of Isoniazid Resistance in Mycobacterium bovis Bacillus CalmetteGue'rin used for Immunotherapy in Bladder Cancer. Clinical Infectious Diseases, 52,8688.

Wright, E.L., quenelle, D.C., suling, W.J., and barrow, W.W. 1996. Use of Mono Mac 6 Human Monocytic Cell Line and J774 Murine Macrophage Cell Line in Parallel Antimycobacterial Drug Studies. Antimicrobial agents and chemotherapy, 2206-2208.

$\mathrm{Xu}$, S., Cooper, A., Sturgill-Koszycki, S., van Heyningen, T., Chatterjee, D., Orme, I., Allen, P. and Russell, D.G. 1994. Intracellular trafficking in Mycobacterium tuberculosis and Mycobacterium aviuminfected macrophages. J Immunol, 153, 2568-78. 